\title{
General and Specific Combining Ability Studies in Single Cross Hybrids of Maize (Zea mays L.)
}

\author{
P. Roopa Sowjanya*, E. Gangappa and S. Ramesh
}

Department of Genetics and Plant Breeding, UAS, GKVK, Bengaluru - 560065, India

*Corresponding author

Keywords

Maize, Combining ability, GCA, SCA, Hybrids

\section{Article Info}

Accepted: 04 September 2019 Available Online: 10 October 2019

\section{A B S T R A C T}

A set of sixteen inbred lines were evaluated to assess the ability of inbred lines, they were crossed in half diallel mating design. A total of 120 single cross hybrids were developed and evaluated in simple lattice square design at $\mathrm{K}$ block experimental plots of Department of Genetics and Plant Breeding, University of Agricultural Sciences, Bengaluru. The observations were recorded on five randomly selected plants. Analyses of variances revealed that, all the hybrids were differed significantly for the traits under consideration which indicates the presence of genetic variability. The SCA variance was higher than the GCA variance, indicates the presence of both additive and non-additive gene action controlling the grain yield and its attributing traits. The inbreds with positive significant GCA can be used as parents in hybrid development program, whereas, the hybrid combinations with significant SCA have to evaluate across different locations to know its genetic potential.

\section{Introduction}

Maize is the world's most widely grown cereal and is the primary staple food in many developing countries. However, the average productivity of Indian cultivars is half of the world average productivity Anon. (2017), to increase the productivity of maize in India breeders has to concentrate on developing high heterotic hybrids, to develop hybrids identification of potential inbreds are more crucial. Further identification of potential inbreds, first step is to dissect the combing abilities of inbred lines. The concept of general and specific combining ability was introduced by Sprague and Tatum (1942) and its mathematical modeling was set about by Griffing (1956) in his classical paper in conjunction with the diallel crosses. The value of any population depends on its potential per se and its combining ability in crosses Vacaro et al., (2002). The usefulness of these concepts for the characterization of an inbred in crosses have been increasingly popular among the 
maize breeders since the last few decades. The variances of general and specific combining ability are related to the type of gene action involved. Variance for GCA includes additive portion of total variance arising largely from dominance and epistatic deviations (Rojas and Sprague, 1952; Jenkins et al., 1932).

Maize is a highly cross pollinated crop and there is a wide scope for exploitation of hybrid vigour. Already this phenomenon has been successfully exploited and still there is tremendous potential to exploit several high yielding hybrids and composites.

An understanding of the genetic architecture of parent, their mode of inheritance will greatly aid the breeder to device appropriate breeding methodology to incorporate the traits in question. Diallel analysis is one of the methods employed by which the genetic architecture of a given character, the combing ability and heterosis could be understood. The objective of this study was to evaluate the performance of 16 inbred lines developed by University of Agricultural Sciences, GKVK, Bengaluru. These promising lines were never appeared to be tested before for their breeding potential per se in specific combinations (sca) and their overall performance in crosses (gca).

\section{Materials and Methods}

The experimental material in the present investigation comprised of sixteen inbred lines selected from previous studies Pavan (2015). These selected lines were crossed following diallel mating design at K-block experimental plots of Department of Genetics and Plant Breeding, University of Agricultural Sciences, GKVK, Bengaluru, during kharif2015.

Each entry was raised in one row with a row length of $3 \mathrm{~m}$ and the spacing maintained was $60 \mathrm{~cm}$ between the rows and $30 \mathrm{~cm}$ between the plants. The recommended packages of practices were followed to raise a good crop. The observation on grain yield and its important component traits were recorded from five competitive plants which were selected randomly from each treatment to record observations on days to 50 percent tasseling, days to 50 percent silking, anthesis to silking interval, plant height $(\mathrm{cm})$, ear length $(\mathrm{cm})$, ear circumference $(\mathrm{cm})$, number of kernel rows ear ${ }^{-1}$, number of kernels row $^{-1}$, rind weight, 100- grain weight (g) and grain yield $(\mathrm{g})$. The mean values of these five plants were used for combining ability analysis (Diallel) as per the method suggested by Hayman (1954).

\section{Results and Discussion}

In the present investigation, the analysis of variance for combining ability in respect to 11 quantitative characters under study is presented in Table 1. Analysis of variance revealed that all the lines chosen were highly significant for all the traits indicating sufficient variability. The mean sum of squares for hybrids were highly significant for all the traits indicating sufficient variability, whereas, variance among the inbredswas highly significant for all the traits except for anthesis to silking interval.

The variance due to the interaction effect of parents verses hybrids was also found highly significant for cob length, cob width, kernel row $\mathrm{cob}^{-1}$, kernels row ${ }^{-1}$, plant height and 100 grain weight indicates that the parents chosen were diverse and with a difference genetic background. The SCA variance was higher than the GCA variance indicating predominance of non-additive variance. Nonadditive variance was greater than additive variance for all the character indicating the predominance of non-additive gene action. These results are in confirmative with the findings of Lay and Razdan (2017) and Aminu et al., (2014) and Zhang et al., (2016). 
The general combining ability effects calculated for each parent are presented in Table 2, among sixteen inbreds evaluated for eleven characters gave evidence that inbred lines MQPM 43, MAI 315, MAI 386 and M 2 were the best combiners for grain yield, apart from grain yield, 18816 has showed positive significant gca effects for days to anthesis, days to silking, number of kernels row ${ }^{-1}$, cob width found to be good combiners for yield attributing traits. In contrast MAI 137, MAI 754, and MAI 387 were exhibited negative significant gca effects for grain yield. MQPM 43, MAI 315, MAI 386, M2, MAI 157 and 18816 can be used as inbreds in future breeding program. The gca effects is a value derived from the general mean of hybrid involving all parents. The gca effects of parents may be positive or negative. Simmonds (1979) pointed out that the gca values were relative and dependent upon the mean of the chosen material. It is better to choose parents possessing significant gca effects or merely based on mean performance. This assumption is based on the principle that gca effect reflects additive gene action.

Sometimes, the immediate hybrid may not perform well despite both the parents possessing high gca effects for a trait, due to interaction of the parental gca effects which may cause distortions on expectationNepir et al.,(2015). The reverse trend may also happen with low performing parents showing high hybrid values than expected similar results observed by Aung et al.,(2016).

This interaction is measured by the sca effects of the hybrids. In the present study estimates of specific combining ability of 120 crosses for the eleven characters are presented in Table 3. The crossesnamely.MAI $157 \times$ MAI 315 followed by MAI $315 \times$ MAI 360, MAI $327 \times$ MAI 386, MAI $360 \times$ MAI 754, M $2 \times$ MAI 187, MAI $157 \times$ MAI 386, $18816 \times 757$ and $18816 \times 18701$ exhibited positive significant sca effects for grain yield. M2 x 18701 was found as good combiner for days to silking, anthesis to silking interval and 100 seed weight with significant positive sca effects. MAI $327 \times$ MAI 757, MAI $360 \times$ MAI 175 found to be good combination for cob width with siginificant sca effects, MAI $315 \times$ MAI 387, MAI $360 \times$ MAI 175, 18816 $\times$ MAI 387 observed as good combiner for ear length, the crosses MAI $157 \times$ MAI 387 and MAI $315 \times$ MQPM 43 were found to be good combination for kernels rows per ear and 100 seed weight which exhibited positive significant sca effects. But all the traits under study are under predominance of additive and non additive gene effects which are in same line with the results of Abuali et al., 2012;Pavan et al., 2011.

Two factors are considered important for the evaluation of an inbred line in the production of hybrid maize; characteristics of the line itself and behavior of the line in a particular hybrid combination. As performance of hybrid can be subdivided into two categories i.e., general and specific combining ability Rojas and Sprague (1952), superiority of a line on the basis of combining ability estimates can only be decided precisely after knowing the purpose of a certain breeding programme whether, it is to develop high yielding OPV's (open pollinated varieties) or the superior combinations of hybrids. Of the inbred lines evaluated, MAI 386 followed by MQPM 43, M2, MAI 315 and MAI 157 have the greatest values of gca effects, considering the existence of greater additive gene action, these inbreds can be used as inbred lines in future breeding program.

Crosses namely, MAI $157 \times$ MAI 315 followed by MAI $315 \times$ MAI 360, MAI $327 \times$ MAI 386, MAI $360 \times$ MAI 754, M $2 \times$ MAI 187 showed high positive significant of sca are adequate for developing high-yielding maize hybrids. 
Table. 1 ANOVA of $16 \times 16$ half diallel crosses and their parents for grain yield and its component traits in maize

\begin{tabular}{|c|c|c|c|c|c|c|c|c|c|c|c|c|}
\hline & df & D T A & D T S & A S I & $\mathbf{E} \mathbf{L}$ & $\mathbf{E ~ W}$ & K R & K P R & P H & $\mathbf{R} \mathbf{W}$ & G W & $100 \mathrm{GW}$ \\
\hline Replications & 1.00 & $37.80 * * *$ & $61.56 * * *$ & 2.88 & 1.26 & 0.56 & 0.05 & 1.13 & $575.94 *$ & 28.99 & 2152.69 & 57.45 \\
\hline Parents + Hybrids & 135.00 & $7.68 * * *$ & $8.82 * * *$ & $1.371 * * *$ & $9.75 * * *$ & $2.03 * * *$ & $32.87 * * *$ & $3.70 * * *$ & $832.46 * * *$ & $70.51 * * *$ & $2048.80 * * *$ & $55.77 * * *$ \\
\hline Parents & 15.00 & $9.06 * * *$ & $9.96 * * *$ & 0.36 & $8.02 *$ & $2.58 * * *$ & $47.34 * * *$ & $5.13 * * *$ & $1023.14 * * *$ & $56.07 * *$ & $1895.80 * *$ & $64.81 * * *$ \\
\hline Hybrids & 119.00 & $7.54 * * *$ & $8.74 * * *$ & $1.48 * * *$ & $9.85 * * *$ & $1.95 * * *$ & $30.48 * * *$ & $3.37 * * *$ & $808.15 * * *$ & $72.48 * * *$ & $2080.05 * * *$ & $54.05 * * *$ \\
\hline $\begin{array}{c}\text { Parent Vs. } \\
\text { Hybrids }\end{array}$ & 1.00 & 0.10 & 1.90 & 2.89 & $23.56^{*}$ & $3.75 *$ & $100.14^{*}$ & $20.84 * * *$ & $865.80 * *$ & 52.51 & 625.38 & $123.77 *$ \\
\hline Error & 135.00 & 1.81 & 2.27 & 0.75 & 4.47 & 0.70 & 15.47 & 1.12 & 102.56 & 21.91 & 847.77 & 20.93 \\
\hline Total & 271.00 & 4.86 & 5.75 & 1.07 & 7.09 & 1.37 & 24.09 & 2.41 & 467.91 & 46.15 & 1450.89 & 38.42 \\
\hline GCA & 15.00 & $4.49 * * *$ & $5.12 * * *$ & $1.22 * * *$ & $9.86 * * *$ & $1.39 * * *$ & $24.36 * * *$ & $3.00 * * *$ & $794.92 * * *$ & $72.94 * * *$ & $1867.03 * * *$ & $51.52 * * *$ \\
\hline SCA & 120.00 & $3.74 * * *$ & $4.32 * * *$ & $0.61 * *$ & $4.25 * * *$ & $0.97 * * *$ & $15.44 * * *$ & $1.70 * * *$ & $368.89 * * *$ & $30.54 * * *$ & $919.07 * * *$ & $24.93 * * *$ \\
\hline Error & 135.00 & 0.91 & 1.13 & 0.37 & 2.23 & 0.35 & 7.74 & 0.56 & 51.28 & 10.96 & 423.88 & 10.46 \\
\hline GCA/SCA ratio & - & 0.01 & 0.06 & 0.19 & 0.20 & 0.09 & 0.11 & 0.11 & 0.13 & 0.17 & 0.16 & 0.15 \\
\hline D T A & \multicolumn{4}{|c|}{ Days to anthesis (days) } & K R & : & \multicolumn{3}{|c|}{ Kernels rows ear $^{-1}$} & & & \\
\hline D T S & \multicolumn{4}{|c|}{ Days to silking (days) } & K P R & : & \multicolumn{3}{|c|}{ Kernels row ${ }^{-1}$} & & & \\
\hline A S I & \multicolumn{4}{|c|}{ Anthesis to silking interval } & P H & : & \multicolumn{3}{|c|}{ Plant height $(\mathrm{cm})$} & & & \\
\hline $\mathbf{E} \mathbf{L}$ & \multicolumn{4}{|c|}{ Ear length $(\mathrm{cm})$} & R W & : & \multicolumn{3}{|c|}{ Rind weight (g) } & & & \\
\hline E W & \multicolumn{4}{|c|}{ Ear Width $(\mathrm{cm})$} & G W & : & \multicolumn{3}{|c|}{ Grain weight (g) } & & & \\
\hline $100 \mathrm{G} \mathrm{W}$ & \multicolumn{3}{|c|}{100 grain weight $(\mathrm{g})$} & & Df & : & \multicolumn{3}{|c|}{ Degrees of freedom } & & & \\
\hline
\end{tabular}


Table.2 Estimates of general combining ability of 16 inbreds for grain yield and its component traits in maize

\begin{tabular}{|c|c|c|c|c|c|c|c|c|c|c|c|}
\hline Inbreds & DTA & D T S & A S I & $\mathbf{E} \mathbf{L}$ & $\mathbf{E} \mathbf{W}$ & K R & K P R & P H & R W & G W & $100 \mathrm{GW}$ \\
\hline MAI 137 & $0.71 * *$ & $1.14 * * *$ & $0.43 * *$ & $-1.46 * * *$ & $-0.40 * *$ & $-1.35^{*}$ & $-0.42 *$ & 0.00 & $-4.78 * * *$ & $-14.51 * *$ & -1.21 \\
\hline MAI 157 & 0.01 & -0.37 & $-0.38 * *$ & $-1.03 * *$ & 0.04 & -0.75 & -0.19 & $10.62 * * *$ & -0.59 & 5.60 & $1.76^{*}$ \\
\hline MAI 315 & $0.48 *$ & 0.14 & $-0.34 *$ & 0.12 & 0.17 & 0.96 & $-0.72 * * *$ & $10.42 * * *$ & $2.95 * * *$ & $12.17 *$ & $2.26^{* *}$ \\
\hline MAI 327 & -0.35 & -0.29 & 0.07 & -0.06 & 0.01 & -0.68 & 0.18 & $4.37 * *$ & -0.81 & 1.72 & 0.34 \\
\hline MAI 360 & -0.27 & 0.05 & $0.31 *$ & -0.13 & 0.08 & -0.10 & 0.24 & $-9.72 * * *$ & 0.70 & 5.12 & $1.48 *$ \\
\hline 754 & $0.60 * *$ & 0.20 & $-0.41 * *$ & -0.52 & $-0.51 * * *$ & -1.00 & -0.31 & $-6.52 * * *$ & $-2.30 * *$ & $-13.63 * *$ & $-1.60 *$ \\
\hline 18758 & 0.36 & 0.28 & -0.08 & 0.21 & $-0.34^{*}$ & -0.17 & 0.17 & -1.98 & -0.36 & $-9.97 *$ & 0.51 \\
\hline 18816 & $0.66^{* *}$ & $0.66^{* *}$ & 0.00 & -0.41 & $0.33^{*}$ & -1.02 & $0.77 * * *$ & $-8.62 * * *$ & -0.30 & $-12.3 * *$ & 0.37 \\
\hline M 2 & 0.06 & -0.02 & -0.08 & 0.07 & $0.28 *$ & -0.17 & $-0.43^{*}$ & -2.27 & $1.74^{*}$ & $11.63 *$ & $2.39 * *$ \\
\hline MAI 175 & $-0.59 * *$ & -0.29 & $0.30^{*}$ & -0.12 & -0.15 & -0.33 & $0.51 * *$ & $-8.41 * * *$ & -0.59 & 1.70 & $1.48^{*}$ \\
\hline MAI 386 & $-1.10 * * *$ & $-1.38 * * *$ & $-0.27 *$ & $1.16 * * *$ & 0.04 & $2.33 * * *$ & $0.44^{*}$ & -1.03 & $2.34 * *$ & $11.85^{*}$ & -0.35 \\
\hline MAI 387 & -0.10 & 0.02 & 0.12 & -0.43 & $-0.29 *$ & $-1.96^{* *}$ & -0.31 & $-5.11 * *$ & $-1.88 *$ & $-14.79 * *$ & $-2.76^{* * *}$ \\
\hline MQPM 43 & -0.04 & 0.00 & 0.04 & $0.92 * *$ & $0.39 * *$ & 0.74 & -0.01 & $3.86^{*}$ & $1.59 *$ & $12.61 * *$ & 0.73 \\
\hline 747 & 0.21 & 0.14 & -0.07 & 0.10 & $0.31^{*}$ & $1.65^{*}$ & $0.38 *$ & $6.12 * * *$ & $2.34 * *$ & 2.56 & $-2.07 * *$ \\
\hline 757 & -0.20 & 0.08 & $0.28 *$ & 0.14 & -0.04 & 0.84 & -0.11 & 1.23 & 0.90 & 4.32 & -0.71 \\
\hline 18701 & $-0.44^{*}$ & -0.36 & 0.09 & $1.43 * * *$ & 0.09 & 1.02 & -0.18 & $7.02 * * *$ & -0.96 & -4.04 & $-2.62 * * *$ \\
\hline CD for GCA & $* * *$ & $* * *$ & $* * *$ & $* * *$ & $* * *$ & $* * *$ & $* * *$ & $* * *$ & $* * *$ & $* * *$ & $* * *$ \\
\hline CD at $95 \%$ & $0.676 * * *$ & $0.756 * * *$ & $0.434 * * *$ & $1.062 * * *$ & $0.42 * * *$ & $1.976 * * *$ & $0.531 * * *$ & $5.088 * * *$ & $2.352 * * *$ & $14.628 * * *$ & $2.298 * * *$ \\
\hline CD at $99 \%$ & $0.935 * * *$ & $1.046 * * *$ & $0.6 * * *$ & $1.468 * * *$ & $0.581 * * *$ & $2.732 * * *$ & $0.734 * * *$ & $7.034 * * *$ & $3.251 * * *$ & $20.223 * * *$ & $3.177 * * *$ \\
\hline D T A & \multicolumn{4}{|c|}{\begin{tabular}{l|l}
$:$ & Days to anthesis (days)
\end{tabular}} & K R & \multicolumn{3}{|c|}{ Kernels rows ear ${ }^{-1}$} & & & \\
\hline D T S & \multicolumn{4}{|c|}{ Days to silking (days) } & K P R & \multicolumn{3}{|c|}{ Kernels row $^{-1}$} & & & \\
\hline A S I & \multicolumn{4}{|c|}{ Anthesis to silking interval } & P H & \multicolumn{3}{|c|}{ Plant height $(\mathrm{cm})$} & & & \\
\hline $\mathbf{E} \mathbf{L}$ & \multicolumn{4}{|c|}{ Ear length $(\mathrm{cm})$} & R W & \multicolumn{3}{|c|}{ Rind weight (g) } & & & \\
\hline $\mathbf{E} \mathbf{W}$ & \multicolumn{4}{|c|}{ Ear Width $(\mathrm{cm})$} & G W & \multicolumn{3}{|c|}{ Grain weight (g) } & & & \\
\hline $100 \mathrm{G} \mathrm{W}$ & \multicolumn{4}{|c|}{100 grain weight $(\mathrm{g})$} & Df & \multicolumn{3}{|c|}{ Degrees of freedom } & & & \\
\hline
\end{tabular}


Table.3 Estimates of specific combining ability effects of 120 single cross hybrids of maize for grain yield and its components

\begin{tabular}{|c|c|c|c|c|c|c|c|c|c|c|}
\hline Hybrids & DTA & D T S & A S I & P H & $\mathbf{E} \mathbf{L}$ & $\mathbf{E ~ W}$ & N K R & K P R & $\mathbf{R} \mathbf{W}$ & G W \\
\hline MAI $137 \times$ MAI 157 & 1.05 & 1.75 & 0.70 & -10.29 & -0.12 & 0.73 & 2.65 & -0.73 & -17.43 & $2-.302$ \\
\hline MAI $\times$ MAI 315 & -0.51 & 0.84 & $1.35^{*}$ & $-13.58^{*}$ & -1.55 & $-1.78^{* *}$ & -3.97 & -1.39 & $-45.40 *$ & 1.20 \\
\hline MAI $137 \times$ MAI 327 & $-3.28 * * *$ & -1.84 & $1.44^{*}$ & $-14.04 *$ & 0.66 & -0.03 & 2.77 & -0.50 & -9.15 & -1.39 \\
\hline MAI $137 \times$ MAI 360 & 0.93 & 0.62 & -0.31 & $20.35^{* *}$ & -0.37 & -0.41 & -0.91 & 0.44 & 26.75 & -3.03 \\
\hline MAI $137 \times$ MAI 754 & -0.84 & -0.53 & 0.32 & -5.14 & 2.07 & -0.31 & 4.40 & -0.01 & -6.25 & 1.06 \\
\hline MAI $137 \times 18758$ & $-2.89 * *$ & -1.91 & 0.99 & -4.18 & 2.19 & 0.57 & $7.25^{* *}$ & -0.29 & -22.56 & 0.95 \\
\hline MAI $137 \times 18816$ & $-1.99 *$ & $-2.59^{*}$ & -0.59 & 0.96 & -0.39 & 0.25 & $-5.28 *$ & -1.39 & -25.73 & $6.08^{*}$ \\
\hline MAI 137 × M 2 & $-1.79^{*}$ & $-3.70 * * *$ & $-1.91 * *$ & -2.40 & 1.90 & 0.29 & 0.56 & 0.82 & 5.23 & 3.56 \\
\hline MAI $137 \times$ MAI 175 & -1.44 & -1.64 & -0.19 & 7.85 & 1.42 & -0.23 & -1.68 & $1.96^{* *}$ & 27.77 & -6.03 \\
\hline MAI $137 \times$ MAI 386 & 0.77 & 0.65 & -0.12 & 11.16 & -2.11 & -0.64 & -3.94 & 0.14 & -19.29 & -1.19 \\
\hline MAI $137 \times$ MAI 387 & $2.36 * *$ & $2.45^{*}$ & 0.09 & -2.55 & -0.27 & -0.39 & 0.65 & -0.11 & -9.54 & -2.28 \\
\hline MAI $137 \times$ MQPM 43 & 1.21 & 0.98 & -0.23 & 2.77 & -2.47 & $-2.26 * * *$ & -2.85 & -0.91 & -27.45 & $-8.27 * *$ \\
\hline MAI $137 \times 747$ & 1.36 & 1.74 & 0.38 & -4.29 & -2.25 & -0.10 & -2.16 & 0.89 & 3.91 & 4.03 \\
\hline MAI $137 \times 757$ & $2.56 * *$ & $3.39 * *$ & 0.82 & 6.10 & -0.89 & -0.29 & -0.94 & $-1.40^{*}$ & 17.25 & -2.33 \\
\hline MAI $137 \times 18701$ & 1.41 & 0.74 & -0.68 & 8.81 & -2.34 & 0.48 & -3.03 & -0.54 & 7.11 & 3.59 \\
\hline MAI $157 \times$ MAI 315 & -0.81 & -0.04 & 0.77 & $-13.71^{*}$ & -0.65 & 0.40 & -0.57 & $2.07 * *$ & $65.08 * *$ & -2.78 \\
\hline MAI $157 \times$ MAI 327 & $-2.28 *$ & $-2.91 * *$ & -0.64 & -2.17 & -1.12 & $-1.14 *$ & -1.03 & -0.73 & -26.06 & $-6.35^{*}$ \\
\hline MAI $157 \times$ MAI 360 & -1.77 & $-2.15^{*}$ & -0.39 & $-20.56 * *$ & 0.80 & $-1.15^{*}$ & 2.49 & -0.79 & $-39.75^{*}$ & -1.00 \\
\hline MAI $157 \times$ MAI 754 & -0.54 & -0.20 & 0.34 & 1.74 & 1.14 & 0.98 & -0.61 & -0.44 & -21.56 & 4.09 \\
\hline MAI $157 \times 18758$ & -1.50 & -1.19 & 0.31 & 3.20 & -2.29 & -0.68 & $-7.04 * *$ & 0.48 & -2.87 & 2.98 \\
\hline MAI $157 \times 18816$ & 1.00 & 1.03 & 0.03 & -12.67 & 0.78 & -0.76 & -2.79 & 0.27 & -1.84 & -1.39 \\
\hline MAI $157 \times$ M 2 & $-1.89^{*}$ & $-2.18^{*}$ & -0.29 & 6.48 & 0.25 & -0.27 & -0.34 & 0.28 & -14.48 & -0.41 \\
\hline MAI $157 \times$ MAI 175 & 0.06 & 0.29 & 0.23 & $16.62 *$ & -0.66 & 0.67 & -1.08 & 0.33 & -7.14 & 2.00 \\
\hline MAI $157 \times$ MAI 386 & $1.86^{*}$ & $2.17^{*}$ & 0.31 & $22.74 * *$ & -1.14 & -0.07 & $-5.84^{*}$ & 0.40 & -22.90 & 1.84 \\
\hline MAI $157 \times$ MAI 387 & -0.04 & -0.13 & -0.09 & $25.82 * * *$ & 1.08 & 0.80 & $5.35 *$ & 1.00 & 29.95 & $8.75 * *$ \\
\hline MAI $157 \times$ MQPM 43 & 1.41 & 1.10 & -0.31 & $14.35 *$ & -0.70 & -0.12 & 2.75 & $-1.74^{*}$ & $45.54 *$ & -1.25 \\
\hline
\end{tabular}




\begin{tabular}{|c|c|c|c|c|c|c|c|c|c|c|}
\hline MAI $157 \times 747$ & $-1.84 *$ & $-2.34 *$ & -0.50 & -11.92 & 1.14 & -0.15 & 3.04 & -0.74 & -18.00 & 1.56 \\
\hline MAI $157 \times 757$ & 1.57 & 1.71 & 0.15 & -3.02 & 2.58 & 0.45 & $6.65^{*}$ & -1.14 & 19.54 & 2.20 \\
\hline MAI $157 \times 18701$ & 0.81 & 0.36 & -0.46 & 7.19 & -0.98 & -0.24 & -0.33 & -0.97 & 16.80 & $-6.38^{*}$ \\
\hline MAI $315 \times$ MAI 327 & $-2.14 *$ & -1.93 & 0.22 & 7.04 & 1.35 & 0.75 & 2.66 & -1.00 & -0.04 & 1.14 \\
\hline MAI $315 \times$ MAI 360 & -0.04 & -0.07 & -0.03 & $13.63^{*}$ & 2.15 & 0.96 & $8.67 * *$ & -1.06 & $59.96 * *$ & 2.50 \\
\hline MAI 315 × MAI 754 & -0.31 & -0.31 & -0.01 & 7.44 & 0.19 & 0.89 & -3.42 & -0.21 & 16.56 & 0.59 \\
\hline MAI $315 \times 18758$ & -0.86 & -0.60 & 0.27 & -2.80 & 0.35 & 0.90 & 2.25 & -0.69 & -4.15 & 1.48 \\
\hline MAI $315 \times 18816$ & 0.64 & -0.88 & $-1.51 * *$ & 5.14 & 1.18 & 0.26 & 3.80 & $-1.69 *$ & 35.98 & 1.61 \\
\hline MAI $315 \times$ M 2 & 0.54 & 0.61 & 0.07 & -9.82 & -1.36 & -0.02 & -1.35 & -0.49 & $-43.25^{*}$ & -3.41 \\
\hline MAI $315 \times$ MAI 175 & 0.59 & 1.08 & 0.48 & $16.32 *$ & 1.09 & 0.50 & 1.91 & -0.44 & 13.38 & 3.00 \\
\hline MAI $315 \times$ MAI 386 & $2.20^{*}$ & $2.36^{*}$ & 0.16 & $16.94 *$ & -1.34 & 0.15 & -1.56 & $-1.66^{*}$ & -28.88 & 2.84 \\
\hline MAI $315 \times$ MAI 387 & 1.40 & 1.36 & -0.03 & $40.52 * * *$ & $4.05 * *$ & 0.89 & 2.34 & 0.89 & 32.88 & -0.25 \\
\hline MAI $315 \times$ MQPM 43 & -0.46 & -1.11 & -0.66 & $15.15^{*}$ & 1.05 & 1.10 & -0.56 & $1.58 *$ & 15.76 & 2.75 \\
\hline MAI $315 \times 747$ & -0.01 & -0.95 & -0.94 & $-24.21 * * *$ & -1.25 & -0.50 & -1.87 & 1.29 & -12.88 & -2.94 \\
\hline MAI $315 \times 757$ & $3.20 * * *$ & $2.50^{*}$ & -0.70 & $-69.32 * * *$ & $-2.97 *$ & $-1.88 * * *$ & $-6.05^{*}$ & -1.11 & $-53.53 * *$ & $-10.80 * * *$ \\
\hline MAI $315 \times 18701$ & $-2.25^{*}$ & $-2.35^{*}$ & -0.10 & $-23.710 * * *$ & -1.37 & -0.14 & 0.06 & 1.11 & 8.33 & -3.39 \\
\hline MAI $327 \times$ MAI 360 & 0.30 & 0.05 & -0.24 & 3.68 & -0.97 & -0.98 & -4.38 & 0.44 & -11.18 & -4.58 \\
\hline MAI $327 \times$ MAI 754 & 1.43 & 1.81 & 0.38 & -1.02 & $-3.28 *$ & 0.07 & -4.58 & 0.49 & -13.68 & $-6.49 *$ \\
\hline MAI $327 \times 18758$ & $2.36 * *$ & 1.83 & -0.54 & $14.94^{*}$ & 2.48 & -0.53 & $8.98 * * *$ & $-1.791 *$ & 29.01 & 3.89 \\
\hline MAI $327 \times 18816$ & 0.07 & -0.36 & -0.43 & $26.07 * * *$ & 0.20 & -0.28 & 3.84 & -0.90 & 3.14 & 0.53 \\
\hline MAI $327 \times$ M 2 & -0.33 & 0.13 & 0.46 & $19.22 * *$ & 2.67 & -0.44 & $5.69 *$ & 0.91 & -12.60 & -4.50 \\
\hline MAI $327 \times$ MAI 175 & 1.13 & 0.70 & -0.43 & 9.47 & -2.08 & -0.35 & $-5.55^{*}$ & -0.64 & 12.64 & 1.92 \\
\hline MAI $327 \times$ MAI 386 & 1.44 & 1.89 & 0.45 & $21.98 * *$ & $2.88 *$ & $1.157 *$ & 2.78 & $-1.96 * *$ & $53.28 * *$ & $9.25^{* *}$ \\
\hline MAI $327 \times$ MAI 387 & -0.37 & -0.91 & -0.54 & 8.07 & 0.98 & 0.64 & 1.78 & 0.48 & 33.63 & 0.17 \\
\hline MAI $327 \times$ MQPM 43 & $-2.72 * *$ & $-3.69 * * *$ & -0.97 & 12.60 & -0.98 & $1.51 * *$ & 1.68 & 0.58 & 17.02 & 1.67 \\
\hline MAI $327 \times 747$ & -1.58 & -0.83 & 0.75 & $-39.67 * * *$ & 0.05 & 0.23 & 5.07 & $-1.51^{*}$ & 23.78 & 3.98 \\
\hline MAI $327 \times 757$ & 0.34 & 1.53 & $1.19 *$ & 3.72 & -0.70 & $2.08 * * *$ & -2.22 & 1.09 & -11.38 & 3.61 \\
\hline MAI $327 \times 18701$ & $1.88^{*}$ & 1.77 & -0.11 & $-31.06 * * *$ & $-3.14 *$ & -0.19 & -1.50 & $2.85 * * *$ & $-46.41 *$ & -3.97 \\
\hline MAI $360 \times$ MAI 754 & -0.26 & 0.07 & 0.33 & 1.59 & 0.39 & $1.23 *$ & 5.04 & $2.12 * *$ & $51.82 * *$ & $14.86 * * *$ \\
\hline
\end{tabular}




\begin{tabular}{|c|c|c|c|c|c|c|c|c|c|c|}
\hline MAI $360 \times 18758$ & 0.68 & 1.29 & 0.61 & 13.04 & 2.75 & 0.66 & 1.30 & 0.35 & 4.81 & -0.75 \\
\hline MAI $360 \times 18816$ & $2.78 * *$ & $4.70 * * *$ & $1.92 * *$ & $18.67 * *$ & -1.68 & 0.64 & $-7.44 * *$ & 0.24 & -16.26 & -5.11 \\
\hline MAI $360 \times$ M 2 & 1.39 & $2.09 *$ & 0.71 & 6.32 & -0.11 & 0.93 & 0.41 & 1.05 & -20.50 & 1.86 \\
\hline MAI $360 \times$ MAI 175 & $2.73 * *$ & $2.15^{*}$ & -0.58 & $28.46 * * *$ & $2.99 *$ & $1.227 *$ & 1.16 & $1.49 *$ & 30.34 & -1.72 \\
\hline MAI $360 \times$ MAI 386 & $3.54 * * *$ & $3.44 * * *$ & -0.10 & 3.59 & $-2.91 *$ & -0.06 & -1.30 & 0.37 & 28.29 & -4.89 \\
\hline MAI $360 \times$ MAI 387 & $-5.65 * * *$ & $-6.35 * * *$ & -0.69 & $-35.32 * * *$ & -1.05 & -0.53 & $-6.90 * *$ & -1.28 & $-38.76^{*}$ & 1.03 \\
\hline MAI $360 \times$ MQPM 43 & -1.71 & $-2.33^{*}$ & -0.62 & -8.80 & 0.10 & $-1.44 *$ & 1.50 & -0.78 & 13.13 & 2.03 \\
\hline MAI $360 \times 747$ & $-1.96^{*}$ & -1.87 & 0.10 & $-21.07 * *$ & -1.23 & $-1.63 * *$ & -1.12 & $-1.57^{*}$ & $-50.01 *$ & -2.66 \\
\hline MAI $360 \times 757$ & $-2.15^{*}$ & $-2.31^{*}$ & -0.16 & -4.18 & 0.17 & -0.68 & 3.70 & -1.07 & $-48.67 *$ & -1.03 \\
\hline MAI $360 \times 18701$ & 0.99 & 1.23 & 0.24 & -5.97 & -1.62 & $-1.11 *$ & 2.32 & $-1.70^{*}$ & -10.41 & -0.11 \\
\hline MAI754 $\times 18758$ & $-2.59 * *$ & -1.76 & 0.83 & -3.65 & -2.20 & 0.63 & -1.29 & $1.798 *$ & 4.91 & -2.16 \\
\hline MAI754 $\times 18816$ & $-3.69 * * *$ & $-2.44^{*}$ & $1.25^{*}$ & -3.52 & -0.08 & $-2.05 * * *$ & 2.36 & -0.71 & -14.56 & 4.98 \\
\hline MAI754 × M 2 & $-4.08 * * *$ & $-4.55 * * *$ & -0.47 & $-22.87 * * *$ & -2.14 & -0.91 & $-5.98^{*}$ & -0.80 & -32.10 & -3.55 \\
\hline MAI754 $\times$ MAI 175 & $-2.13^{*}$ & $-2.78 * *$ & -0.65 & 3.27 & -0.27 & 0.01 & 4.87 & 1.25 & 34.34 & 4.36 \\
\hline MAI754 $\times$ MAI 386 & $2.87 * *$ & $2.50 *$ & -0.37 & 2.19 & 2.40 & 0.75 & 3.11 & 0.32 & 29.19 & 1.70 \\
\hline MAI754 $\times$ MAI 387 & $5.36 * * *$ & $5.20 * * *$ & -0.17 & $-27.02 * * *$ & -2.51 & $-1.22 *$ & $-8.09 * *$ & -0.33 & -1.16 & $-8.88 * *$ \\
\hline MAI754 $\times$ MQPM 43 & $2.11^{*}$ & 1.13 & -0.99 & 10.00 & -0.01 & 0.19 & 1.60 & -1.23 & 8.03 & 1.11 \\
\hline MAI754 $\times 747$ & 1.16 & 1.09 & -0.08 & $21.73 * *$ & 2.06 & -0.60 & 3.89 & $-1.42 *$ & 6.18 & $8.92 * *$ \\
\hline MAI754 × 757 & 1.28 & 0.94 & -0.33 & 7.63 & 2.77 & 0.35 & 3.21 & 1.08 & -18.28 & -2.44 \\
\hline MAI754 × 18701 & $2.51 * *$ & $2.38 *$ & -0.13 & $-25.16^{* * * *}$ & 2.12 & 0.10 & -0.78 & 0.15 & -16.51 & $-10.02 * *$ \\
\hline $18758 \times 18816$ & $2.35^{*}$ & $2.37 *$ & 0.02 & -7.06 & $-5.51 * * *$ & $-1.87 * *$ & $-7.87 * *$ & $-1.58 *$ & $-48.66^{*}$ & -2.64 \\
\hline $18758 \times$ M 2 & 0.26 & 0.76 & 0.51 & 5.59 & -2.50 & -0.62 & -0.63 & $1.42 *$ & -19.51 & $-6.16^{*}$ \\
\hline $18758 \times$ MAI 175 & $-2.99 * *$ & $-3.16^{* *}$ & -0.18 & $14.52 *$ & -1.92 & 0.43 & -3.17 & -0.43 & 7.93 & -4.25 \\
\hline $18758 \times$ MAI 386 & -1.08 & -1.18 & -0.10 & -3.15 & 1.06 & 0.53 & 1.37 & 0.84 & 20.88 & -2.41 \\
\hline $18758 \times$ MAI 387 & 0.31 & 0.12 & -0.19 & 11.44 & 0.01 & $-1.14 *$ & -2.14 & 0.50 & 30.23 & 1.50 \\
\hline $18758 \times$ MQPM 43 & -1.44 & -1.76 & -0.32 & -6.04 & 0.96 & -0.09 & $-5.83^{*}$ & -1.31 & 7.71 & -3.50 \\
\hline $18758 \times 747$ & 0.31 & 0.10 & -0.21 & $-23.81 * * *$ & 1.43 & 0.19 & -3.55 & $1.79 *$ & 8.17 & 0.81 \\
\hline $18758 \times 757$ & 0.52 & -0.34 & -0.86 & $-16.91^{*}$ & -1.57 & -1.00 & -2.73 & 0.40 & -18.49 & 2.95 \\
\hline $18758 \times 18701$ & 1.06 & 0.90 & -0.16 & -8.70 & 1.24 & 0.52 & 0.38 & 1.27 & 5.18 & 2.86 \\
\hline
\end{tabular}




\begin{tabular}{|c|c|c|c|c|c|c|c|c|c|c|}
\hline $18816 \times$ M 2 & -0.54 & -0.62 & -0.08 & $16.22 *$ & -0.48 & 0.51 & -0.77 & 0.11 & 14.72 & -3.53 \\
\hline $18816 \times$ MAI 175 & 0.01 & -0.15 & -0.16 & $-22.63 * *$ & -1.28 & -0.07 & 1.88 & -0.89 & -29.44 & 4.39 \\
\hline $18816 \times$ MAI 386 & 0.22 & 0.04 & -0.18 & $-21.01 * *$ & -1.39 & 0.31 & 1.32 & -1.07 & $-38.89^{*}$ & 1.73 \\
\hline $18816 \times$ MAI 387 & 0.31 & 0.04 & -0.28 & $-60.92 * * *$ & $2.98 *$ & 0.62 & -0.98 & $-1.813 *$ & -30.55 & $8.14 * *$ \\
\hline $18816 \times$ MQPM 43 & 0.26 & 0.36 & 0.10 & -5.90 & -1.92 & $2.112 * * *$ & 3.32 & $1.484^{*}$ & 14.34 & 5.14 \\
\hline $18816 \times 747$ & -0.29 & -1.18 & -0.89 & $27.82 * * *$ & -0.21 & 0.86 & 0.21 & -0.41 & 31.80 & -0.55 \\
\hline $18816 \times 757$ & -1.48 & -1.03 & 0.46 & 10.72 & 0.50 & 0.87 & -0.28 & -0.31 & $44.54 *$ & 4.09 \\
\hline $18816 \times 18701$ & $2.46 * *$ & $2.52 *$ & 0.06 & $16.93^{*}$ & -0.31 & $1.22 *$ & 0.74 & 0.46 & $48.50^{*}$ & 4.50 \\
\hline M $2 \times$ MAI 175 & 0.21 & 0.04 & -0.18 & -4.99 & -0.90 & 0.49 & 1.33 & -0.23 & 0.92 & $9.86^{* *}$ \\
\hline M $2 \times$ MAI 386 & 0.63 & 1.03 & 0.40 & 9.13 & -1.58 & 0.74 & 0.07 & $-1.55^{*}$ & -2.34 & 2.70 \\
\hline M $2 \times$ MAI 387 & 0.12 & 0.03 & -0.09 & -0.78 & 2.40 & $1.13^{*}$ & $6.26^{*}$ & 0.40 & $47.714^{*}$ & 1.61 \\
\hline M $2 \times$ MQPM 43 & 0.66 & 0.15 & -0.52 & -5.76 & -0.41 & 0.42 & -2.73 & -0.31 & -3.40 & 5.11 \\
\hline M $2 \times 747$ & 1.01 & 1.41 & 0.40 & -0.53 & 0.32 & -0.06 & 1.66 & 0.20 & 16.46 & $7.42 *$ \\
\hline M $2 \times 757$ & 1.32 & 0.46 & -0.86 & $-37.13 * * *$ & 0.27 & $-2.88 * * *$ & -3.93 & $-2.20 * *$ & 26.30 & -0.94 \\
\hline M $2 \times 18701$ & $3.36 * * *$ & $4.80 * * *$ & $1.44^{*}$ & -2.42 & 0.18 & $-1.20 *$ & $-6.61 *$ & $-2.43 * * *$ & $-46.33 *$ & $8.47 * *$ \\
\hline MAI $175 \times$ MAI 386 & -0.30 & -0.13 & 0.15 & 1.20 & -1.83 & -6.96 & -1.27 & -22.22 & 3.49 & 4.61 \\
\hline MAI $175 \times$ MAI 387 & 1.27 & 2.26 & 0.96 & -1.56 & -1.59 & -0.84 & -0.75 & -51.28 & -6.57 & -5.70 \\
\hline MAI $175 \times$ MQPM 43 & -1.05 & 0.03 & 0.95 & 1.10 & 0.61 & 6.33 & 0.52 & -0.80 & 4.73 & -1.28 \\
\hline MAI $175 \times 747$ & 0.77 & 1.54 & 0.65 & 0.14 & -0.41 & -1.77 & -1.39 & -1.71 & -1.56 & -2.08 \\
\hline MAI $175 \times 757$ & -1.57 & -1.62 & -0.19 & 1.80 & 0.19 & 5.30 & 1.80 & 9.24 & 3.13 & -3.96 \\
\hline MAI $175 \times 18701$ & -2.08 & -1.61 & 0.42 & -2.65 & -0.99 & -3.86 & -1.36 & 8.16 & -4.33 & -3.35 \\
\hline MAI 386 × MAI 387 & $-3.32 * * *$ & $-5.11 * * *$ & $-1.79 * *$ & 2.97 & 0.24 & 0.37 & 0.26 & 0.31 & 22.59 & 2.86 \\
\hline MAI $386 \times$ MQPM 43 & -1.27 & -1.19 & 0.07 & 1.00 & 0.00 & 0.90 & 0.86 & $3.81 * * *$ & 27.98 & $-6.63 *$ \\
\hline MAI $386 \times 747$ & $-1.92 *$ & -0.53 & $1.39 *$ & -0.26 & 1.93 & 0.46 & -1.24 & $2.92 * * *$ & 24.04 & -1.33 \\
\hline MAI $386 \times 757$ & $-3.01 * *$ & $-2.78 * *$ & 0.23 & -10.37 & -0.21 & -0.23 & -2.23 & $-1.87 * *$ & -9.81 & 3.30 \\
\hline MAI $386 \times 18701$ & $2.13^{*}$ & $2.06^{*}$ & -0.06 & 13.04 & -2.63 & -0.11 & 0.28 & 0.39 & 28.14 & 2.72 \\
\hline MAI $387 \times$ MQPM 43 & 1.51 & 1.30 & -0.21 & $28.69 * * *$ & -0.99 & 0.26 & -3.44 & -0.53 & -0.46 & 5.78 \\
\hline MAI $387 \times 747$ & 1.56 & 0.76 & -0.80 & 9.82 & -1.92 & -0.82 & -2.05 & -0.82 & $-51.00 * *$ & -5.41 \\
\hline MAI $387 \times 757$ & -0.92 & $2.02 *$ & $2.94 * * *$ & $-15.68 *$ & -2.09 & 0.20 & -2.43 & 1.17 & $-42.16 *$ & $-9.77 * *$ \\
\hline
\end{tabular}


Int.J.Curr.Microbiol.App.Sci (2019) 8(10): 313-323

\begin{tabular}{|c|c|c|c|c|c|c|c|c|c|c|}
\hline MAI $387 \times 18701$ & $-2.27 *$ & 0.26 & $2.54 * * *$ & -5.47 & $-3.21 *$ & -0.67 & -1.62 & -0.45 & $-55.50 * *$ & -5.35 \\
\hline MQPM $43 \times 747$ & -0.68 & 1.18 & $1.87 * *$ & -13.25 & 0.07 & -0.97 & -1.65 & 1.21 & -8.51 & -5.41 \\
\hline MQPM $43 \times 757$ & 0.62 & 0.94 & 0.31 & 8.04 & $-3.42 *$ & -0.59 & $-5.63^{*}$ & -0.62 & $-47.05^{*}$ & $8.72 * *$ \\
\hline MQPM $43 \times 18701$ & 0.56 & 1.48 & 0.918 & $-17.14 *$ & $10.58 * * *$ & 0.13 & 1.47 & -0.76 & -26.01 & $8.142 * *$ \\
\hline $747 \times 757$ & -0.42 & -0.59 & -0.17 & $31.87 * * *$ & 1.03 & 0.42 & 0.65 & 0.37 & 20.18 & -0.96 \\
\hline $747 \times 18701$ & 0.41 & -1.55 & $-1.97 * * *$ & 1.58 & -0.29 & -0.44 & -1.13 & 0.24 & 7.24 & -0.05 \\
\hline $757 \times 18701$ & $-3.67 * * *$ & $-4.29 * * *$ & -0.62 & $20.67 * *$ & 0.15 & 0.01 & 3.08 & -0.55 & 3.08 & 1.08 \\
\hline CD@95\% & 1.78 & 1.99 & 1.14 & 13.41 & 2.80 & 1.10 & 5.21 & 1.4 & 38.57 & 6.06 \\
\hline CD@ 99\% & 2.35 & 2.63 & 1.51 & 17.73 & 3.70 & 1.46 & 6.89 & 1.85 & 51.00 & 8.01 \\
\hline D T A & : & \multicolumn{3}{|c|}{ Days to anthesis (days) } & K R & : & \multicolumn{3}{|c|}{ Kernels rows ear ${ }^{-1}$} & \\
\hline D T S & : & \multicolumn{3}{|c|}{ Days to silking (days) } & K P R & : & \multicolumn{3}{|c|}{ Kernels row $^{-1}$} & \\
\hline A S I & : & \multicolumn{3}{|c|}{ Anthesis to silking interval } & P H & : & \multicolumn{3}{|c|}{ Plant height $(\mathrm{cm})$} & \\
\hline $\mathbf{E} \mathbf{L}$ & : & \multicolumn{3}{|c|}{ Ear length $(\mathrm{cm})$} & $\mathrm{R} \mathrm{W}$ & : & \multicolumn{3}{|c|}{ Rind weight (g) } & \\
\hline $\mathbf{E} \mathbf{W}$ & $:$ & \multicolumn{3}{|c|}{ Ear Width $(\mathrm{cm})$} & G W & : & \multicolumn{3}{|c|}{ Grain weight (g) } & \\
\hline $100 \mathrm{G} \mathrm{W}$ & : & \multicolumn{3}{|c|}{100 grain weight $(\mathrm{g})$} & Df & : & \multicolumn{3}{|c|}{ Degrees of freedom } & \\
\hline
\end{tabular}




\section{Acknowledgement}

The senior author acknowledges the financial support from University Grant Commission (UGC) in the form of Rajiv Gandhi National Fellowship (RGNF) for pursuing Ph.D. programme.

\section{References}

Abuali, A. I., Abdelmulla, A. A.,Khalafalla, M. M., Idris, A. E. and Osman, A. M., 2012. Combining ability and heterosis for yield and yield components in maize (Zea mays L.), Aust. J. Basic \& Appl. Sci., 6 (10): 36-41.

Aminu, D., Dawadu, M. A. and Modu, A., 2014. Combining ability and heterosis for different agronomic characters in maize (Zea mays L.) under drought stress in the Sudan savanna of Borno State, Nigeria., J. Plant Breed. Crop. Sci., 6 (10): 128134.

Anonymous, 2017.World Agricultural Production.Foreign Agricultural Service, United States Department of Agriculture, Washington, DC, 13p.

Aung, N., Khaing,T. T., Than, H. and Zar,M. T., 2016.Evaluation of hybrid maize (Zea mays L.) performance crossing within inbreds developed by composite line selection method. J. Agric. Res., 3 (1): 47-54.

Griffing, B., 1956. Concept of general and specific combining ability in relation to diallel crossing system. Australian J. of Biological Sciences 9(4): 463-493.

Hayman, B. I., 1954. The Theory and analysis of diallel crosses. Genetics, 39: 789-809.

Jenkins,M. T. and Brunaon, A. M., 1932.A method of testing inbred line of maize in cross bed combinations, J. Ann. Sci. Agron., 24: 523-530.
Lay, P. and Razdan, A. K., 2017. Combining ability analysis of morpho-physiological characters in maize (Zea mays L.) inbred lines, Int. J. Curr. Microbiol. App. Sci., 6 (4): 470-476.

Nepir, G., Wegary, D. and Zeleke,H., 2015. Heterosis and combining ability of highland quality protein maize inbred lines, Maydica, 60:1-12.

Pavan, R., Prakash, G. and Mallikarjuna, N. M., 2011.General and specific combining ability studies in single cross hybrids of maize (Zea mays L.), Current Biotica 5(2): 196-208.

Pavan M. P., 2015, Identification of high yielding and nutrient rich maize (Zea mays. L). M.Sc. (Agri.) Thesis, Univ. Agric. Sci., Bengaluru.

Rojas, B. A. and Sprague, G. F., 1952.A comparison of variance components in corn yield trials: III general and specific combining ability and their interaction with location and years. Agron. J., 44: 462-6.

Simmomds, N. W., 1979.Principles of crop improvement, Longman Group Ltd., London, pp.110-116.

Sprague, G. P. and Tatum, L. S., 1942.General vs specific combining ability in six crosses of corn, J. Amer. Soc.Agron.34: 923-932.

Vacaro, E., Barbosa, N. J. F., Pegoraro, D. G., Nuss, C. N. and Conceicao, L. D. H., 2002.Combining ability of twelve maize populations. Pesuisagropecuria Brasilerira, Brasília, 37: 67-72.

Zhang, Y.D., Fan, X. M., Yao, W., Piepho, H. P. and M.S. Kang, 2016.Diallel analysis of four maize characters and a modified heterosis hypothesis. Crop Sci., 56:1115-1126.

\section{How to cite this article:}

Roopa Sowjanya, P., E. Gangappa and Ramesh, S. 2019. General and Specific Combining Ability Studies in Single Cross Hybrids of Maize (Zea mays L.). Int.J.Curr.Microbiol.App.Sci. 8(10): 313323. doi: https://doi.org/10.20546/ijcmas.2019.810.032 